\title{
Trends in Decayed Teeth among Middle-Aged and Older Adults in the United States: \\ Socioeconomic Disparities Persist Over Time
}

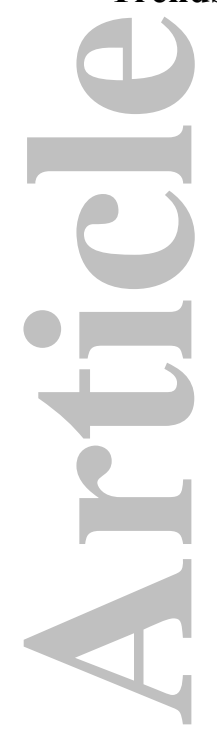

This is the author manuscript accepted for publication and has undergone full peer review but has not been through the copyediting, typesetting, pagination and proofreading process, which may lead to differences between this version and the Version record. Please cite this article as doi:10.1111/jphd.12153. 


\begin{abstract}
OBJECTIVES: While trends in tooth loss among older adults have been well documented and show a decline over the last few decades, little is known about trends in tooth decay which may lead to tooth loss. The study aim was to examine trends in tooth decay among adults ages 50 years and older in the U.S. and determine whether these trends were consistent across demographic and socioeconomic subgroups of middle-aged and older adults.

METHODS: Secondary analysis of data collected through detailed oral health examinations in the National Health and Nutrition Examination (NHANES) surveys 1988-1994 and 1999-2004. Tooth decay was measured as active caries. Multivariable associations were estimated using negative binomial regression models.

RESULTS: Averaged over time, the mean number of decayed teeth was 0.54 . Rates of decay remained stable over time. Males, Non-Hispanic Blacks, Mexican-Americans and those of Other race/ethnicity as well as those with fewer years of education and lower levels of income had more decayed teeth. The increased number of decayed teeth for Mexican-Americans and those of Other race/ethnicity was due in part to differing levels of education and income. Trends over time did not vary by any of these demographic and socioeconomic characteristics. Trends in the number of decayed teeth did not meaningfully change when the numbers of missing and filled teeth were controlled.

CONCLUSIONS: Although studies have shown the number of middle-aged and older Americans experiencing tooth loss has decreased over time, trends in tooth decay have remained relatively stable, with socioeconomic disparities persisting over time.
\end{abstract}

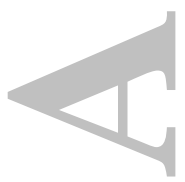

Key Words: tooth decay; trends; oral health 


\section{INTRODUCTION}

Tooth decay is prevalent among middle-aged and older adults. Reports from the 1999-2004 NHANES indicated that among adults in the U.S. ages 50 to 64 years, the prevalence of untreated tooth decay was $11.0 \%$, while for those ages 65 or older, the prevalence was estimated to be over $17 \%$ (1). This reported increase in the prevalence of active tooth decay with age corresponds to an observed increase in tooth loss with age (1).

0

There is considerable evidence that across all age groups the distribution of decay is skewed, with a higher risk of untreated caries or caries experience (DMFT: decayed, missing or filled teeth) concentrated among those with a lower socioeconomic position (determined by one's own or parental educational or occupational background, or income), particularly in developed countries (2). Social determinants of health inequalities in general health have been well documented (3). These inequalities in oral health have also been documented, and gradients by income and education observed in oral health are similar to gradients seen in general health, implying commonalities of the social determinants of both oral and general health (4).

While tooth decay is related to preventable causes such as sugar consumption, fluoride usage, and access to dental care, disparities exist even when dental care is available, due in part to social determinants of health (5). In the U.S., adults with fewer than 12 years of education and lower income have not only higher rates of one or more decayed teeth but also lower restoration rates (6). Similar results have been reported from Denmark (7). Higher levels of income and education are likely associated with preventive means and care such as greater availability of toothpaste and floss, less sugar in the diet, health behaviors including increased frequency of flossing and cleaning, and more dental service utilization and restorative treatment resulting in less untreated tooth decay (2). Sabbah and colleagues reported clear socioeconomic disparities in health behaviors related to oral health. After adjusting for these health behaviors, however, the association between socioeconomic indicators and oral health attenuated but did not disappear (8). As 
previous studies indicated, the determinants of health disparities are complex. Other factors such as quality of dental care, oral health knowledge and behaviors, and the dental workforce could also affect oral health outcomes (9-11). Education and income are independent predictors of oral health and are only moderately correlated, making preventive measures more complicated (12).

Research has also focused on the distribution of tooth decay by demographic variables such as age, sex, and race/ethnicity. In the ElderSmile program in Manhattan, no differences by level of education or race/ethnicity were noted. Older men, however, had more decayed teeth than women (13). Recent research has shown individual patterns of tooth decay are linked to distinct risk factors such as age, sex, race, and educational attainment as well as oral health behaviors such as tooth brushing (14). Wu and colleagues recently reported that among adults ages 60 or older, Blacks and Mexican-Americans had significantly higher numbers of decayed teeth than Whites, when age, race, sex, education, income, marital status, health behaviors, health status, dental care utilization and coverage by dental insurance were controlled (11).

Few studies have examined trends in the number of decayed teeth over time. Brown and colleagues, using data from the NHANES studies conducted from 1974-1994, reported that among adults 18 to 45 years of age the number of untreated caries declined by 50 percent (15). It is not known if this trend would be observed among middle-aged and older adults. In a study of Australian public dental patients ages 18 and older conducted in 1995/1996 and again in 2001/2002, the opposite trend was observed: the number of decayed teeth increased over time (16). Thirty-year trends in the prevalence of dental caries among adults ages 20-80 were reported from Sweden. There was a steady decrease in the mean number of decayed/filled teeth among those ages 20-50 years. Among those in the 60-80 years age groups, however, the percentage of decayed/filled teeth increased during the same time period (17). Bernabé and colleagues examined age, period, and cohort effects in the number of DMFT over time. Period and cohort effects were small, but there was a large increase in DMFT with increasing age. That is, despite recent 
declines in caries among children, levels of decay increased with age (18). For these latter two studies, results were not available for decayed teeth alone.

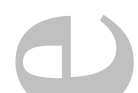

While social inequalities in the prevalence of decayed teeth have been noted, for example in relation to oral hygiene (19), trends in tooth decay across diverse demographic and socioeconomic subgroups of middle-aged and older adults have not been fully documented. Studies have focused on younger populations or across all age groups or have combined decayed teeth with missing and filled teeth. Given the complexity of oral health in older adults, instead of using a composite index of DMFT, it is also important to evaluate oral health using separate indicators of the number of missing teeth, the number of filled teeth, and the number of decayed teeth. Filled teeth represent access to care which may change the findings regarding decayed teeth if the indicators are summed. Also, older adults have a significantly higher number of missing teeth than decayed or filled teeth. Using a summary index to measure oral health would be heavily focused on missing teeth. Trends in decayed teeth alone have been less studied. Using data from the Piedmont Dental Study, a longitudinal six-year study of 810 older adults who were dentate at baseline, Liang and colleagues reported that the number of decayed teeth decreased over time. Relative to Whites, older Black participants had more decayed teeth averaged over time, but there were no racial differences in the rate of change over time (20).

The purpose of this study was to examine trends in the number of decayed teeth over time among middleaged and older adults in the U.S., and to determine whether any observed changes were consistent across demographic and socioeconomic subgroups of the population. We chose to focus on decayed teeth alone, taking advantage of the unique data provided by the NHANES clinical oral health examination to focus on one outcome independent of the others. Untreated decay can only be assessed through oral examination. We hypothesized that the number of decayed teeth among middle-aged and older adults would decrease during the period from 1988-1994 to 2004. We also hypothesized that individuals with fewer years of education and lower levels of income would have a slower decrease in the number of 
decayed teeth over time, and that education and income would partially explain any racial/ethnic differences in the rate of change over time. We hypothesized that these trends would be significant independent of the numbers of filled and missing teeth.

\section{METHODS}

Study Sample

Data from the NHANES conducted from 1988-1994 and 1999 to 2004 were used for these trend analyses.

The NHANES surveys are administered regularly to nationally representative samples of the noninstitutionalized population of the U.S. The survey design has been well documented (21). A multistage area probability design was used to select eligible households. A subset of eligible participants was selected to participate in detailed medical and oral health examinations. The analyses in this study focused on dentate adults age 50 years and older who participated in the oral health examinations in NHANES III conducted 1988-1994 (n=4,568), NHANES 1999-2000 (n=1,358), NHANES 2001-2002 $(n=1,588)$ and NHANES 2003-2004 $(n=1,599)$ for a total of 9,113 participants. Some NHANES oral health data are available for years after 2004, but the dental examination did not include measures of the number of decayed teeth. Because the NHANES was not a longitudinal study, the samples analyzed here represent a repeated cross-sectional design.

\section{Study Variables}

The detailed oral health examinations were conducted by licensed trained dentists. According to NHANES documentation (22), tooth surfaces affected by dental caries were identified using modified Radike's criteria (23), with the modification being the elimination of the 'extraction indicated' code. The dental examiners used a non-magnifying mirror and a dental explorer to examine each tooth surface for caries. Pits and fissures were coded as carious if the explorer caught after insertion with moderate pressure and there was accompanying softness at the base of the tooth, opacity adjacent, or evidence of undermining enamel (22). Tooth decay referred to caries into dentine and cavitated only. Evidence of 
decayed teeth was reported by each of the four (incisors and canines) or five (molars) tooth surfaces. The unit of analysis for this manuscript was the tooth. Third molars were excluded from these analyses so the possible number of decayed teeth could range from 0 to 28 . It is important to note these counts represent the number of teeth with untreated decay among the number of permanent teeth present. Because of the cross-sectional nature of the NHANES, the dental examiner could not determine if the decay was active or arrested. The NHANES clinical criteria used to identify dental caries were consistent from 1988-1994 and 1999-2004.

Demographic variables included age, race, sex and years of education as recorded in the NHANES questionnaire. For these analyses, age was dichotomized to reflect ages 50-64 (code 0) and ages 65 years and older (code 1). Race was coded as White Non-Hispanic (code 0), Black Non-Hispanic (code 1), Mexican-Americans (code 2) and Other race/ethnicity (code 3). Other race/ethnicity included Asians, Native Americans and Hispanics whose country of origin was not Mexico. Those groups classified as Other were not sampled in sufficient numbers to allow population estimates. Prior to 2007/2008, the only Hispanics oversampled were Mexican-Americans and reliable estimates for 'All Hispanics' could not be derived. Hispanics whose country of origin was not Mexico, therefore, were included in the 'Other' category.

Five levels of education were coded: $<9$ years of education (code 0$), 9-11$ years of education (code 1), high school graduate (code 2), some college (code 3) and college or more (code 4). Poverty status was calculated based on the ratio of total household income to the U.S. poverty level computed by the Bureau of Census (21). For consistency across waves, we grouped participants into quartiles based on the distribution among those ages 50 years and older who participated in the oral health examination at the particular wave separately by wave, with Quartile 1 representing those with the lowest values on the Poverty Index and therefore the lowest income. Education and poverty were treated as categorical in the descriptive analyses. These categories, however, had a linear relationship with the number of decayed 
teeth and were treated as continuous in the regression analyses. Time was coded as 0 for NHANES III, 8.5 for NHANES 1999-2000, 10.5 for NHANES 2001-2002 and 12.5 for NHANES 2003-2004, reflecting the midpoint of the number of years following the first round.

\section{Statistical Analyses}

The counts of the number of decayed teeth among dentate participants (those with one or more permanent teeth) were estimated using negative binomial models. As noted by Allison (24), negative binomial models can be estimated for distributions with excess zeros when there are few absolute zeros, as is the case in these data. Like zero-inflated models, the negative regression model allows for overdispersion and often fits better than a zero-inflated model as evaluated by AIC or BIC statistics.

We began with a model controlling only for time (Model 1) and then added age, race and sex as covariates (Model 2). We added product terms (time*age, time*sex and time*race) which were then removed if not significant. Level of education was then added to the initial demographic model as well as a product term for time*education, and the product term was removed if not significant (Model 3). Finally, poverty quartile was added to the model with a product term time* poverty which was removed if not significant (Model 4). As a second set of analyses, we estimated models in the same manner as specified above but controlled for the number of missing teeth and the number of filled teeth. Filled teeth and missing teeth are competing outcomes with decayed teeth.

Wave specific clinical examination sampling weights were used to reflect the characteristics of the U.S. population age 50 years and older at the time of each NHANES survey. All statistical tests were twosided and adjusted to take stratification and clustering effect into account. Significance levels were set at $\mathrm{p}<0.01$ to minimize the probability of a Type I error given the large sample. 
Tests of statistical interactions or whether changes in the number of decayed teeth over time significantly differed by age, race, sex, education or level of poverty were assessed on an additive (effect on the count) scale rather than on a multiplicative (effect on the rate ratio) scale as discussed by Rothman, Greenland and Lash (25) and Mustillo et al. (26). SAS software (Version 9.3) was used for the descriptive analyses. Stata software (Version 12) was used to estimate the negative binomial regression models with the margins post-estimation command used to compute the incidence rate ratio (IRR) for the rate of change in the effect of the demographic and socioeconomic subgroups on the number of decayed teeth.

\section{RESULTS}

The characteristics of the sample are shown in Table 1 . The mean number of decayed teeth was 0.63 in the NHANES III survey (1988-1994) and 0.52 some twelve years later in NHANES 2003-2004.

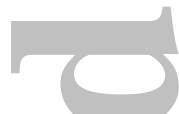

(Table 1 here)

In our first model with time as the only covariate (Table 2, Model 1), the number of decayed teeth among those who were dentate did not change over time $(I R R=0.98, p=0.011)$. In the model controlling for age, sex and race as well as time (Model 2), we found, averaged over time, that men had more decayed teeth than women, and Blacks, Mexican-Americans and those of Other race/ethnicity had more decayed teeth than Whites. Interactions with time and age, sex, and race/ethnicity were not significant. We next added education to the model (Model 3) and found those with fewer years of education had more decayed teeth. As a final step, we added poverty level to the model (Model 4). Those with higher levels of income had fewer decayed teeth. In our final model, (Model 4), we found those ages 50-64 years had more decayed teeth compared to those ages 65 years and older. Men had more decayed teeth than women, and Blacks had more decayed teeth Whites. Mexican-Americans and those of Other race/ethnicity did not significantly differ from Whites when education and income were controlled. Changes in the number of decayed teeth over time did not vary by level of education or income.

(Table 2 here) 
In the second set of analyses we controlled for the number of missing teeth and the number of filled teeth (see Table 3). The number of decayed teeth did not change over time when the number of missing and filled teeth were controlled (Model $1: \mathrm{IRR}=0.98, \mathrm{p}=0.020$ ). The number of missing teeth was significantly associated with the number of decayed teeth ( $I R R=0.98, p<0.0001)$, with those having more missing teeth having fewer decayed teeth. Those who had more filled teeth also had fewer decayed teeth (IRR $=0.86$, $\mathrm{p}<0.0001)$. As shown in Model 2, averaged over time, men, Blacks and Mexican-Americans had more decayed teeth. The effect of time did not vary by age, sex or race.

Averaged over time and controlling for the number of missing teeth and the number of filled teeth, those with more years of education had fewer decayed teeth (Model 3: IRR $=0.85, \mathrm{p}<0.0001$ ). Those with higher levels of income also had fewer decayed teeth (Model 4: IRR $=0.73, \mathrm{p}<0.0001$ ). The effect of time did not vary by level of education or income. In our final model, (Model 4), those ages 50-64 and men had more decayed teeth. Blacks and Mexican-Americans no longer significantly differed from Whites. The increased number of decayed teeth initially observed in Mexican-Americans was due in part to level of education.

\section{(Table 3 here)}

When the number of missing teeth and the number of filled teeth were added to the models as shown in Table 3, the overall observed trends in the number of decayed teeth over time did not meaningfully change. The effect of the number of missing teeth was quite small (IRR=0.98), while the effect of the number of filled teeth was larger (IRR=0.89). Comparing the results of Model 4 as shown in Tables 2 and 3, we can see that the effects of education and race/ethnicity were the most changed when filled teeth were added to the model. That is, part of the effect of education and race/ethnicity is due to the number of filled teeth. For example, in Table 2, Blacks had 80\% more decayed teeth than Whites. In Table 3, Blacks had only $25 \%$ more decayed teeth over time compared to Whites when filled teeth were added to the model. This suggests that Blacks had more decayed teeth than Whites over time because they had fewer 
filled teeth. The disparities noted in Table 2 are reduced but persistent when the number of filled teeth is controlled. Similarly, those with more years of education had fewer decayed teeth as shown in Table 2 $(I R R=0.77)$. When the number of filled teeth was added to the model as shown in Table 3 , the protective effect of education was reduced ( $I R R=0.91$ ), in part because those with more years of education had more filled teeth.

\section{DISCUSSION}

While previous studies have shown among middle-aged and older adults in the U.S. that tooth loss has decreased over time, we did not observe a similar pattern with regard to the number of decayed teeth in this same age group. That is, the data did not support our hypothesis. During the period from 1988-1994 and 1999 to 2004, the mean number of decayed teeth among those ages 50 years and older remained essentially stable. In addition, socioeconomic differences persisted over time, and no specific subgroups saw either an increase or decrease in the number of decayed teeth relative to other subgroups. Our second hypothesis, therefore, was also not supported by the data. These findings are in contrast to our earlier reports for trends in the number of missing teeth which suggested that decreases in tooth loss were primarily obseryed among those with higher incomes (27). Although studies have examined inequalities in decayed teeth over time in other countries such as the United Kingdom and Norway $(28,29)$, this study is one of the first to examine the trends of tooth decay as measured by the number of decayed teeth determined through a clinical oral health examination among American adults across demographic and socioeconomic subgroups over an extended period of time.

While previous research has shown adults are retaining more of their teeth, interventions are needed to maintain healthy teeth free of decay. Improving knowledge and promoting health related behaviors alone would not yield significant results in addressing oral health disparities (8). A broader and more holistic approach is needed to tackle oral health disparities in the U.S. Based on a framework developed by the WHO (30), four levels of policy action can be developed to address social determinants of health 
disparities: 1) Improve social mobility and social benefits to protect vulnerable populations; 2) Improve the availability, accessibility, and affordability of oral health promoting products and services; 3) Develop targeted and tailored interventions that promote individual's healthy lifestyle, coping strategies and social support; and 4) Increase the accessibility of dental care to disadvantaged populations (31).

Adults with fewer educational and economic resources may be less likely to receive adequate dental care.

Health insurance programs such as Medicaid for those with lower incomes cover only limited dental services. Interventions providing an educational component could potentially decrease some of these oral health disparities. Data from Australia, for example, show controlling for sex, place of birth, education, and income that high dental knowledge of tooth decay prevention was associated with fewer decayed teeth and more filled teeth (32) . Data on the trends of tooth loss in the U.S. show socioeconomic disparities in edentulism are decreasing over time, but disparities in missing teeth are increasing (27). Socioeconomic disparities in the number of decayed teeth persist. Decayed teeth are a temporary state. That is, either a decayed tooth is treated and wouldn't be reflected in counts of teeth with untreated decay or the decayed tooth is left untreated and later becomes a missing tooth. This may contribute to the consistency over the study period of the number of decayed teeth and the difference in findings from those of tooth loss.

The findings presented here show persistent disparities in tooth decay between Blacks and Whites over the past two decades, due for the most part to Blacks having fewer filled teeth. Although recently some programs and services have been developed in Black communities addressing oral health disparities (33), more efforts, such as improving access to dental care, quality of dental services, and an increased diverse dental professional workforce are needed to further decrease oral health disparities in this country.

Our study shows that men had more decayed teeth than women. Untreated tooth decay is strongly associated with dental service use, free sugar intake, and oral hygiene. Previous literature suggests that 
women are more likely to engage in better oral hygiene, self-care, and preventive dental service use (34, 35), which some researchers speculate may be related to women's acceptance of help seeking and compliance with treatment regimens and lifestyle changes (36). Thus, one possible reason for this gender difference in tooth decay may result from the individual's health beliefs and health seeking behaviors.

Therefore, targeting health behaviors that vary with gender might be an effective strategy for improving oral health.

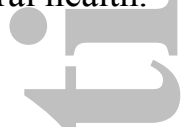

Our research has several limitations. We did not include more recent waves of the NHANES since the number of decayed teeth was not available. The NHANES is not a longitudinal study, so we are measuring change in the prevalence by subgroups rather than at an individual level. We classified education and poverty as continuous rather than ordinal independent variables in our models. Education was nonlinear in the log odds in relation to the number of decayed teeth, while poverty level was linear. Level of education had a monotonic association with the number of decayed teeth. As the years of education increased, however, the number of decayed teeth decreased more rapidly. While these findings of nonlinearity do not affect our conclusions, it is possible that a more comprehensive analysis using categorical measures would detect interactions which we may have missed. Strengths of our research include the use of counts of the number of decayed teeth based on oral health examinations conducted by licensed dentists, the use of representative samples of the older U.S. population over an extended period of time, the associations between socioeconomic disparities and decayed teeth independent of the number of filled and missing teeth, and complex data analysis on trends of tooth decay controlling for demographic characteristics and socioeconomic status.

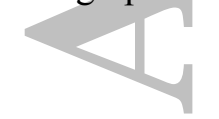

Future research will examine trends in the numbers of treated decayed teeth (filled teeth) over time. It is possible while the number of teeth with untreated tooth decay remains consistent over time that health disparities in the number of filled teeth are actually decreasing. 

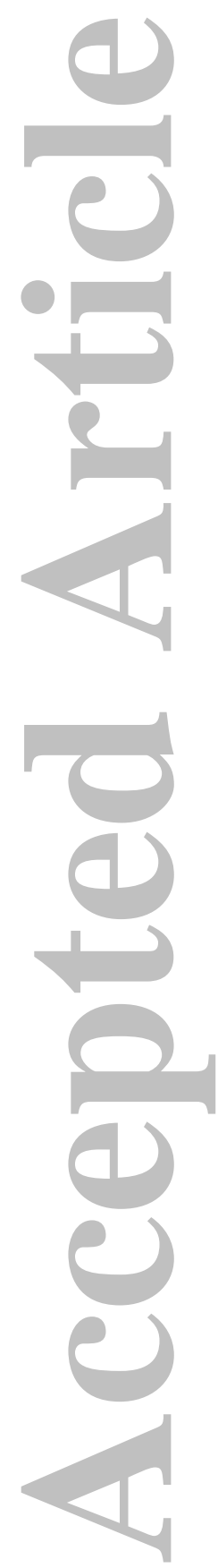

This article is protected by copyright. All rights reserved. 


\section{Acknowledgements}

This study was supported by the National Institute of Dental and Craniofacial Research (NIDCR) through grant R01 DE19110 (Wu). The content is solely the responsibility of the authors and does not necessarily represent the official views of the National Institutes of Health. The authors would like to thank Laurie Barker and Dr. Murray Thomson for their input to the revised manuscript.

Conflict of Interest: The authors did not report any conflicts of interest.

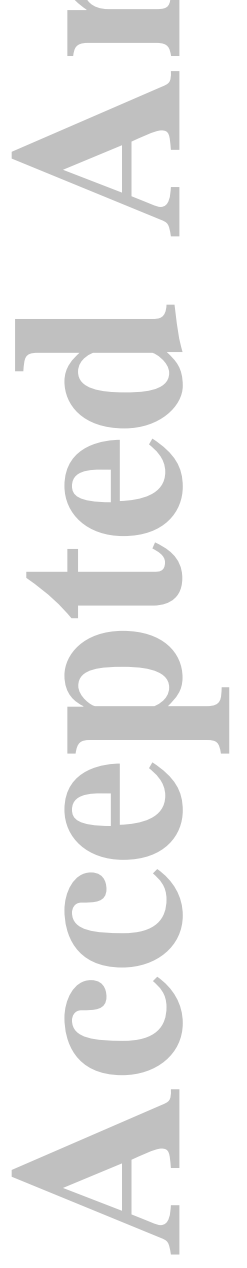




\section{References}

1. Dye B, Tan S, Smith V, Lewis B, Barker L, Thornton-Evans G, et al. Trends in oral health status: United States, 1988-1994 and 1999-2004. National Center for Health Statistics. Vital Health Stat. 2007; $11(248)$.

2. Schwendicke F, Dörfer C, Schlattmann P, Page LF, Thomson WM, Paris S. Socioeconomic inequality and caries: A systematic review and meta-analysis. J Dent Res. 2015; 94:10-8.

3. Marmot M. Social determinants of health inequalities. Lancet. 2005; 365:1099-104.

4. Sabbah W, Tsakos G, Chandola T, Sheiham A, Watt R. Social gradients in oral and general health. J Dent Res. 2007; 86:992-6.

5. Edelstein BL. The dental caries pandemic and disparities problem. BMC Oral Health. 2006; 6 (Suppl 1):S2.

6. Liu Y, Li Z, Walker M. Social disparities in dentition status among American adults. Int Dent J. $2014 ; 64: 52-7$

7. Krustrup U, Holm-Pedersen P, Petersen P, Lund R, Avlund K. The overtime effect of social position on dental caries experience in a group of old-aged Danes born in 1914. J Public Health Dent. $2008 ; 68: 46-52$.

8. Sabbah W, Tsakos G, Sheiham A, Watt R. The role of health-related behaviors in the socioeconomic disparities in oral health. Soc Sci Med. 2009; 68:298-303.

9. Phelan J, Link B. Controlling disease and creating disparities: a fundamental cause perspective. J Gerontol B Psychol Sci Soc Sci. 2005; 60:27-33.

10. Wamala S, Merlo J, Bostrom G. Inequity in access to dental care services explains current socioeconomic disparities in oral health: the Swedish national surveys of public health. J Epidemiol Community Health. 2006; 60:1027-10.

11. Wu B, Liang J, Plassman B, Remle C, Bai L. Oral health among white, black, and MexicanAmerican elders: an examination of edentulism and dental caries. J Public Health Dent. 2011; 71:308-17. 
12. Geyer S, Schneller GS, Micheelis W. Social gradients and cumulative effects of income and education on dental health in the Fourth German Oral Health Study. Community Dent Oral Epidemiol. $2010 ; 38: 120-8$.

13. Northridge M, Ue F, Borrell L, DeLaCruz L, Chakraborty B, Bodnar S, et al. Tooth loss and dental caries in community-dwelling older adults in northern Manhattan. Gerodontology. 2012; 29:e464e73.

14. Shaffer J, Polk D, Feingold E, Wang X, Cuenco K, Weeks D, et al. Demographic, socioeconomic, and behavioral factors affecting patterns of tooth decay in the permanent dentition: principal components and factor analysis. Community Dent Oral Epidemiol. 2013; 41:364-73.

15. Brown L, Wall T, Lazar V. Trends in caries among adults 18 to 45 years old. J Am Dent Assoc. $2002 ; 133: 827-34$.

16. Brennan D, Spencer A. Changes in caries experience among Australian public dental patients between 1995/96 and 2001/02. Aust N Z J Public Health. 2004; 28:542-8.

17. Hugoson A, Koch G. Thirty year trends in the prevalence and distribution of dental caries in Swedish adults (1973-2003). Swed Dent J. 2008; 32:57-67.

18. Bernabe E, Sheiham A. Age, period and cohort trends in caries of permanent teeth in four developed countries. Am J Public Health. 2014; 104:e115-e21.

19. Liang J, Wu B, Plassman B, Bennett J, Beck J. Social stratification, oral hygiene, and trajectories of dental caries among old Americans. J Aging Health. 2014; 26:900-23.

20. Liang J, Wu B, Plassman B, Bennett J, Beck J. Racial disparities in trajectories of dental caries experience. Community Dent Oral Epidemiol. 2013; 6:517-25.

21. Zipf G, Chiappa M, Porter K, Ostchega Y, Lewis B, Dostal J. National health and nutrition examination survey: plan and operations, 1999-2010

Vital Health Stat. 2013; 1:1-37. 
22. Beltrán-Aguilar ED, Barker LK, Canto MT, Dye BA, Gooch BF, Griffin SO, et al. Surveillance for dental caries, dental sealants, tooth retention, edentulism, and enamel fluorosis ---United States, 1988-1994 and 1999-2002. MMWR. 2005; 54:1-44.

23. Radike AW. Criteria for diagnosis of dental caries. Chicago, IL: American Dental Association; 1972.

24. Allison P. Do we really need zero-inflated models? : Statistical Horizons; 2012; Available from: http://statisticalhorizons.com/zero-inflated-models.

25. Rothman K, Greenland S, Lash T. Modern Epidemiology. 3rd ed. Philadephia: Lippincott Williams \& Wilkins; 2008.

26. Mustillo S, Landerman L, Land K. Modeling longitudinal count data: testing for group differences in growth trajectories using average marginal effects. Sociol Methods Res. 2012; 41:467-87. 27. Wu B, Hybels C, Liang J, Landerman L, Plassman B. Social stratification and tooth loss among middle-aged and older Americans from 1988 to 2004. Community Dent Oral Epidemiol. 2014; 42:495502.

28. Holst D, Schuller AA. Equality in adults' oral health in Norway: Cohort and cross-sectional results over 33 years. Comm Dent Oral Epid. 2011; 39:488-97.

29. Steele JG, Treasure ET, O'Sullivan I, Morris J, Murray JJ. Adult Dental Health Survey 2009: Transformations in British oral health 1968-2009. Brit Dent J. 2012; 213:523-7.

30. World Health Organization. A conceptual framework for action on the social determinants of health: Social determinants of health discussion paper 2. Geneva: World Health Organization; 2010. 31. Watt RG. Social determinants of oral health inequalities: implications for action. Comm Dent Oral Epid. 2012; 40(Supp1 2):44-8.

32. Brennan D, Spencer J, Roberts-Thomson K. Dental knowledge and oral health among middleaged adults. Aust N Z J Public Health. 2010; 34:472-5.

33. United States Department of Health and Human Services. Healthy People 2010. Understanding and Improving Health. 2nd ed. Washington, DC: United States Government Printing Office; 2000. 
34. Wiener R, Wu B, Crout R, Plassman B, McNeil D, Wiener M, et al. Hygiene self-care of older adults in West Virginia: effects of gender. J Dent Hyg. 2012; 86:231-8.

35. Vaidya V, Partha G, Karmakar M. Gender differences in utilization of preventive care services in the United States. J Womens Health. 2012; 21:140-45.

36. Green C, Pope C. Gender, psychological factors and the use of medical services: a longitudinal analysis. Soc Sci Med. 1999; 48:1363-72.
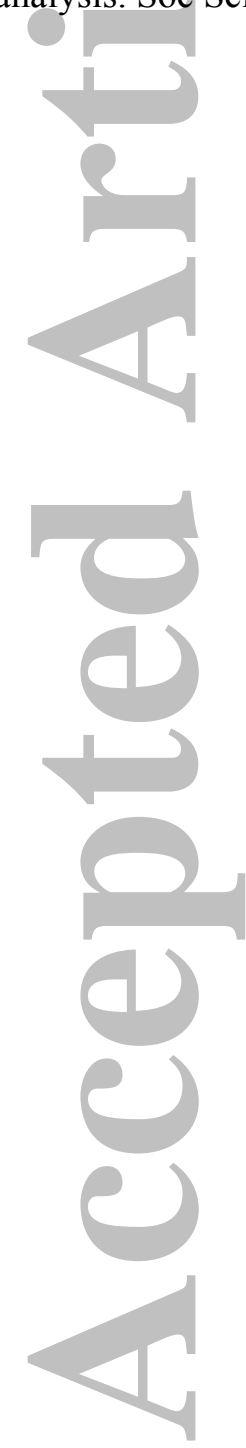
Table 1. Characteristics of the Sample $(n=9113)$

\begin{tabular}{|llr|}
\hline Characteristic & & \\
\hline No. 65 or older & 4617 & $38.5 \%$ \\
No. Female & 4549 & $52.7 \%$ \\
No. White & 5087 & $80.7 \%$ \\
No. Black & 1669 & $8.2 \%$ \\
No. Mexican-American & 1927 & $3.6 \%$ \\
No. Other Race/Ethnicity & 430 & $7.5 \%$ \\
No. $>9$ Yrs Education & 2234 & $9.4 \%$ \\
No. with 9-11 Yrs Education & 1332 & $11.7 \%$ \\
No. with 12 Yrs Education & 2306 & $27.1 \%$ \\
No. with Some College & 1650 & $25.7 \%$ \\
No. with College or More & 1591 & $26.1 \%$ \\
No. in Q 1 (lowest) & 1939 & $12.4 \%$ \\
No. in Q 2 & 2048 \\
No. in Q 3 & 2330 \\
No. in Q4 (highest) & 2796 \\
Mean No. of Decayed Teeth (sd) & 0.54 & $(0.03)$ \\
\hline
\end{tabular}

Numbers are from unweighted data; Means and percentages are weighted to reflect U.S. population age 50 years and older. 
Table 2. Demographic and socioeconomic variables predicting the number of decayed teeth among adults age 50 years and older $1988-2004$ using negative binomial regression models $(\mathrm{n}=9113)$

\begin{tabular}{|c|c|c|c|c|c|c|c|c|}
\hline & \multicolumn{2}{|c|}{ Model 1} & \multicolumn{2}{|c|}{ Model 2} & \multicolumn{2}{|c|}{ Model 3} & \multicolumn{2}{|c|}{ Model 4} \\
\hline Variable & IRR & $\mathrm{p}$-value & IRR & p-value & IRR & p-value & IRR & p-value \\
\hline Intercept & $0.64(0.53,0.76)$ & $<0.001$ & $0.66(0.51,0.85)$ & $<0.001$ & $1.53(1.04,2.25)$ & 0.005 & $2.85(1.82,4.46)$ & $<0.001$ \\
\hline Time & $0.98(0.96,1.00)$ & 0.011 & $0.98(0.95,1.00)$ & 0.019 & $1.00(0.97,1.02)$ & 0.623 & $0.99(0.97,1.01)$ & 0.114 \\
\hline Age 6 & & & $0.88(0.70,1.11)$ & 0.139 & $0.77(0.61,0.96)$ & 0.002 & $0.68(0.52,0.87)$ & $<0.001$ \\
\hline Female & & & $0.68(0.57,0.82)$ & $<0.001$ & $0.65(0.54,0.78)$ & $<0.001$ & $0.62(0.51,0.75)$ & $<0.001$ \\
\hline Race/Ethnicity & & & $2.55(1.95,3.33)$ & $<0.001$ & $2.03(1.56,2.64)$ & $<0.001$ & $1.81(1.36,2.41)$ & $<0.001$ \\
\hline $\begin{array}{l}\text { Mexican- } \\
\text { American }\end{array}$ & & & $2.45(1.63,3.69)$ & $<0.001$ & $1.45(0.98,2.14)$ & 0.014 & $1.27(0.82,1.95)$ & 0.150 \\
\hline Other Race & & & $1.67(1.02,2.72)$ & 0.007 & $1.56(0.92,2.64)$ & 0.028 & $1.32(0.77,2.26)$ & 0.184 \\
\hline Year & & & & & $0.67(0.60,0.75)$ & $<0.001$ & $0.77(0.69,0.86)$ & $<0.001$ \\
\hline $\begin{array}{l}\text { Poverty } \\
\text { Quartile }\end{array}$ & & & & & & & $0.65(0.57,0.74)$ & $<0.001$ \\
\hline
\end{tabular}

$I R R=$ Incidence rate ratio

Confidence limits based on 99\% confidence intervals 
Table 3. Demographic and socioeconomic variables predicting the number of decayed teeth among adults age 50 years and older $1988-2004$ using negative binomial regression models and controlling for the number of missing teeth and the number of filled teeth $(n=9113$ )

\begin{tabular}{|c|c|c|c|c|c|c|c|c|}
\hline & \multicolumn{2}{|l|}{ Model 1} & \multicolumn{2}{|c|}{ Model 2} & \multicolumn{2}{|c|}{ Model 3} & \multicolumn{2}{|c|}{ Model 4} \\
\hline Variable & IRR & p-value & IRR & p-value & IRR & p-value & IRR & p-value \\
\hline Intercept & $2.06(1.48,2.86)$ & 0.020 & $2.07(1.45,2.95)$ & $<0.001$ & $2.75(1.88,4.01)$ & $<0.001$ & $4.18(2.70,6.47)$ & $<0.001$ \\
\hline Time & $0.98(0.96,1.00)$ & 0.020 & $0.98(0.96,1.00)$ & 0.019 & $0.99(0.97,1.01)$ & 0.120 & $0.98(0.96,1.00)$ & 0.027 \\
\hline Age & & & $0.82(0.67,1.02)$ & 0.017 & $0.79(0.63,0.98)$ & 0.006 & $0.73(0.56,0.93)$ & 0.001 \\
\hline Female & & & $0.80(0.67,0.95)$ & 0.001 & $0.77(0.64,0.92)$ & $<0.001$ & $0.73(0.60,0.88)$ & $<0.001$ \\
\hline Race/Ethnicity & & & $1.36(1.07,1.73)$ & 0.001 & $1.31(1.02,1.67)$ & 0.006 & $1.25(0.97,1.62)$ & 0.026 \\
\hline Mexican-American & & & $1.45(1.05,1.99)$ & 0.003 & $1.20(0.87,1.65)$ & 0.137 & $1.10(0.78,1.56)$ & 0.455 \\
\hline Other Race & & & $1.03(0.70,1.52)$ & 0.850 & $1.03(0.69,1.54)$ & 0.846 & $0.95(0.63,1.41)$ & 0.715 \\
\hline Years of Education & & & & & $0.85(0.77,0.92)$ & $<0.001$ & $0.91(0.83,1.00)$ & 0.010 \\
\hline Poverty Quartile & & & & & & & $0.73(0.65,0.82)$ & $<0.001$ \\
\hline $\begin{array}{l}\text { Number of Missing } \\
\text { Teeth }\end{array}$ & $0.98(0.96,0.99)$ & $<0.001$ & $0.98(0.97,1.00)$ & 0.006 & $0.98(0.97,1.00)$ & 0.002 & $0.98(0.97,1.00)$ & 0.001 \\
\hline $\begin{array}{l}\text { Number of Filled } \\
\text { Teeth }\end{array}$ & $0.86(0.83,0.88)$ & $<0.001$ & $0.86(0.84,0.89)$ & $<0.001$ & $0.88(0.85,0.90)$ & $<0.001$ & $0.89(0.86,0.91)$ & $<0.001$ \\
\hline
\end{tabular}

$I R R=$ Incidence rate ratio; Confidence limits based on $99 \%$ confidence intervals 